\title{
The Arabidopsis LRR-RLK, PXC1, is a regulator of secondary wall formation correlated with the TDIF-PXY/TDR-WOX4 signaling pathway
}

Jiehua Wang ${ }^{1}$, Melis Kucukoglu ${ }^{2}$, Linbin Zhang ${ }^{2}$, Peng Chen ${ }^{3}$, Daniel Decker ${ }^{4}$, Ove Nilsson $^{2}$, Brian Jones ${ }^{5}$, Göran Sandberg ${ }^{4}$ and Bo Zheng ${ }^{6^{*}}$

\begin{abstract}
Background: Although a number of leucine-rich repeat receptor-like kinase-encoding genes (LRR-RLKS) have been identified in plants, a functional role has been determined for only a few. Recent studies have demonstrated that an LRR-RLK, PXY/TDR, is important for the process of secondary vascular development. Other studies have indicated that PXY/TDR is unlikely to be the sole LRR-RLK involved in this complex process.

Results: In this study, in silico analyses led to the identification of three Arabidopsis LRR-RLK genes (PXY-correlated; $P X C 1,2,3)$ with transcript accumulation profiles that correlated strongly with several key regulators of vascular development, including PXY/TDR, HB-8, REV, and CLE41. Expression profiling using QPCR and promoter:reporter lines indicated that all three PXC genes are associated with the vasculature. One in particular, PXC1 (At2936570), had a strong correlation with PXY/TDR. Shifting pxc1 mutants from long-days to short-days showed that loss of the gene led to a dramatic reduction in secondary wall formation in xylem fibers. Transcript analysis of mutants for a variety of secondary cell wall-associated genes, including PXY/TDR indicated that the pathways mediated by PXC1 connect with those mediated by the TDIF-PXY/TDR-WOX4 system.

Conclusions: The data indicate that the LRR-RLK, PXC1 is involved in secondary cell wall formation in xylem fibers. Whereas further study is needed to identify the ligands and mode of action of the PXC1 protein, it is clear from this work that similarly to the shoot apical meristem (SAM), secondary vascular development requires contributions from a number of LRR-RLKs.
\end{abstract}

Keywords: LRR-RLK, Arabidopsis, Secondary Wall Formation, TDIF-PXY/TDR-WOX4 Signaling

\section{Background}

LRR-RLKs (leucine-rich repeat receptor-like kinases) comprise the largest group within the RLK (receptor-like kinase) superfamily in plants. Among the more than 400 $R L K$ genes identified in the Arabidopsis genome, over half are LRR-RLKS [1]. The LRR-RLKS can be grouped into 13 subfamilies (I to XIII) [2]. Although the functions of most LRR-RLKs remain undiscovered, it has been suggested that plant LRR-RLKs can be divided into two broad functional categories [2]. That is, some appear to function in plant growth and developmental processes

\footnotetext{
* Correspondence: bo.zheng@mail.hzau.edu.cn

${ }^{6}$ College of Horticulture and Forestry Sciences, Huazhong Agricultural

University, 430070 Wuhan, China

Full list of author information is available at the end of the article
}

such as morphogenesis, organogenesis and hormone signaling, while others appear to be primarily involved in mediating responses to biotic or abiotic stresses and therefore can be said to be defense-related. Some LRRRLKs have been demonstrated to possess dual functions, either through signaling pathway cross-talk or due to their ability to recognize multiple ligands [3]. Well known examples of LRR-RLKs involved in the regulation of plant growth and development are the CLV3 (CLAVATA3)CLV1 (CLAVATA1)-WUS (WUSCHEL) signaling system in the SAM (Shoot Apical Meristem) and the similar, CLE40 (CLAVATA3/ESR40)-CLV1-ACR4 (CRINKLY4)WOX5 (WUSCHEL RELATED HOMEOBOX 5) signaling system in the RAM (Root Apical Meristem) [4-9]. In the SAM, the CLV1 LRR-RLK is essential for maintaining 
a balance between stem cell division and differentiation, and therefore, growth control in the shoot $[6,10,11]$. Loss of CLV1 leads to the accumulation of undifferentiated cells in the SAM. Three other LRR-RLKs that operate in the SAM, the BAM (BARELY ANY MERISTEM) proteins, form a monophyletic group with CLV1, are also involved in maintaining meristem function. Although CLV1 and the BAMs both operate in maintaining SAM function, their expression profiles and functions differ. In contrast to CLV1, the loss of BAM function leads to a reduction in the number of undifferentiated cells [12]. Clearly, LRRRLKs fulfill multiple roles in complex processes such as meristem function.

LRR-RLKs have now been shown to be involved in all three major plant meristems, the SAM and RAM, and the vascular cambium, that produces cells for secondary vascular development. In Arabidopsis, a signaling system consisting of a small CLE peptide, the TDIF (TRACHEARY ELEMENT DIFFERENTIATION INHIBITORY FACTOR), and its receptor PXY/TDR (TDIF RECEPTOR/ PHLOEM INTERCALATED WITH XYLEM) regulates the behavior of vascular stem cells [13]. Genetic analyses showed that at least two pathways diverge early in TDIFPXY/TDR signaling and the WOX4 (WUSCHEL RELATED HOMEOBOX 4), which belongs to the WUS subclade in the WOX family [14], is required for promoting the proliferation of procambial/cambial stem cells but not for repressing their commitment to xylem differentiation in response to the TDIF signal [15]. Correct spatial separation of the expression of the genes encoding PXY/TDR and TDIF, is essential for generating the spatial cues necessary for ordered secondary vascular development[16].

As the only well described pathway so far, the TDIF-PXY/ TDR signal transduction pathway has been suggested to be required both very early in vascular development to orientate the polarity of the vascular bundle, and continuously throughout development to regulate the process [16]. Ectopic expression of TDIF-related genes results in pleiotropic phenotypes including a bushy appearance with small leaves $[17,18]$. Recently, TDIF and CLE42 peptide were found to have an in vivo activity to enhance axillary bud formation and there are indications that PXY/TDR is involved in this process [19]. Together, these results indicate that the TDIF ligand-PXY/TDR signal transduction pathway is an important regulator of multiple developmental processes [19].

Given the size of the LRR-RLK gene family and the evidence of multiple active LRR-RLKs in the SAM, it is likely that other multiple LRR-RLKs are involved in the complex process of secondary vascular development. In this work, we aimed to identify LRR-RLKs other than PXY/TDR that contribute to secondary vascular development through interactions, direct or indirect, with the TDIF-PXY/TDR pathway. Given the importance of the localization of gene expression for PXY/TDR function, we initially performed an in silico co-expression and functional clustering analyses. Three LRR-RLKs (At2g36570, At5g01890 and At2g41820) were identified that had similar transcript profiles to $P X Y / T D R$. We named them $P X C$ for $P X Y / T D R$-correlated genes. Evidence from loss-of -function and gain-of-function analyses showed that PXC1 in particular plays a TDIF-PXY/TDR associated role in the process of secondary cell formation in fiber cells.

\section{Results and discussion}

Co-expression profiling and functional clustering analyses identified three AtLRR-RLKs associated with PXY/TDR

In order to develop our understanding of the functions of AtLRR-RLKs in vascular development, a hierarchical cluster analyses using the microarray data in the Genevestigator database was performed for all AtLRR-RLKS [20]. The dendrogram in Additional file 1 was used to assess transcript profile similarities between the genes. Six out of the 7 genes that clustered with $P X Y / T D R$ exhibited preferential expression in the vasculature, with the highest transcript levels occuring in the stem, apex and floral organs (Figure 1A, 1B). Of the 7, MOL1 (At5g51350, MORE LATERAL GROWTH1), RUL1 (At5g05160, REDUCED IN LATERAL GROWTH1) and VH1 (At2g01950, VASCULAR HIGHWAY1) have already been reported for their vascular functions $[21,22]$. The other three, At2g41820, At5g01890 and $A t 2 g 36570$ are largely uncharacterised. We named the genes as PXC PXY/TDR-correlated genes, PXC1 (At2g36570), PXC2 (At5g01890), and PXC3 (At2g41820). TAIR database information of these three genes indicated that they may be broadly functional. For example, PXC1 displayed a decreased expression level in the Arabidopsis leaves treated by salt [23] and PXC2 appear to be down-regulated in Arabidopsis seedlings under anoxia [24]. For PXC3, its transcription was dramatically repressed in Arabidopsis cell suspensions upon salicylic acid treatment [25]. In an investigation into the roles of LRR-RLKs in Arabidopsis root development, germination of a line mutated in $\mathrm{PXC1}$, $\mathrm{N} 634974$ was shown to be resistant to salt $(200 \mathrm{mM} \mathrm{NaCl})$ and osmotic stress. A $p x c 2$ mutant allele was found to be sensitive to darkness and resistant to osmotic stress treatment (400 mM mannitol) [1]. Together, the results suggest a role for the Arabidopsis PXC genes in defense and other stress-related responses. A putative soybean ortholog of PXC1, GmLRK1 gene has been studied in some detail [26]. Mutating GmLRK1 led to reduced lignification in leaf cells and defective leaf cell elongation. The authors hypothesized that GmLRK1is is involved in the regulation of cell expansion by influencing the development of cell wall architecture [26].

In order to further explore the possibility of a role for the three $P X C$ genes in vasculature development, we developed a putative gene co-regulation network using the 


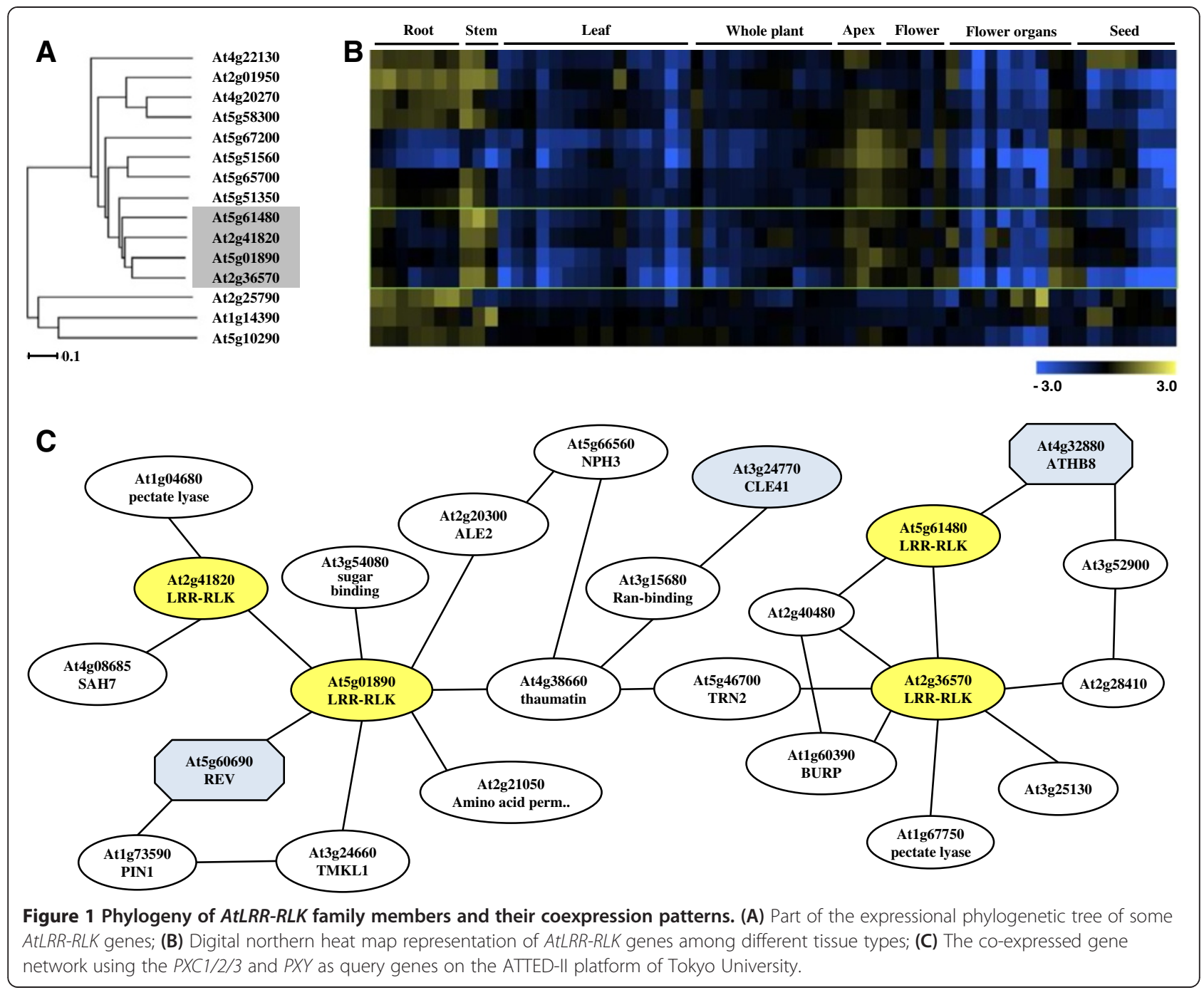

ATTED-II suite of programs (based on publicly available microarray data of 58 experiments, 1388 slides collected by AtGenExpress) [27,28] (Additional file 2). The analyses led to the identification of a putative relationship between PXY/TDR and PXC1 (Figure 1C). The homeobox transcription factor, AtHB8 (HOMEOBOX GENE 8), that is regarded as a procambium and protoxylem cell identity marker, appeared to be similarly associated with $P X Y /$ TDR and PXC1 [29]. PXC1 also correlated strongly with genes encoding enzymes involved in the cell expansion process (Figure $1 C$ ). The $P X C 2$ gene correlated with $P X C 3$ and with REVOLUTA (REVAt5g60690). AtHB8 and REV are Class III HD-ZIP transcriptional regulators and both play important roles in vascular differentiation [30]. CLE41, that encodes the peptide ligand for the PXY/TDR receptor [31], was located between $P X C 1$ and $P X C 2$ in the coexpression network (Figure 1C). The bioinformatic data, therefore, indicated connections between PXY/TDR, and the three PXC proteins in vascular development.

\section{Expression patterns of $P X Y$ and three $P X C$ genes in} vascular tissues

In order to further explore the associations between $P X C 1 / 2 / 3$ and $P X Y / T D R$, we examined their respective expression patterns using a native promoter-driven GUS ( $\beta$-glucuronidase) reporter system. PXY/TDR has been reported to be expressed in the vasculature of a variety of organs including leaves, roots and the stem [16]. Its expression has been shown to be confined to the procambial cells in the developing vascular bundles [16]. The pattern of GUS activity observed in the $p P X Y:: G U S$ was similar to the pattern oberseved in the $p P X C 1:: G U S, p P X C 2:: G U S$, and pPXC3::GUS lines. Specifically, GUS was observed primarily in the vascular strands in cotyledons, the shoot apex, hypocotyls, roots and leaves (Figure 2A-2D, 2F). In the inflorescence stems of the $p P X Y:: G U S$ line, GUS was observed in the protoxylem, procambial cells and in the interfascicular cambial regions (Figure 2E). In the $p P X C 1::$ GUS line, GUS staining was observed primarily in the 
developing xylem of both inflorescence stem and hypocotyl (Figure 2E, Figure 3B). PXC1 expression overlapped with $P X Y$ expression in the basal stem, except that the staining was very faint in protoxylem (Figure 2E). In $p P X C 2:: G U S$ plants, GUS staining in the inflorescence stem was primarily observed in the differentiating vessel cells (Figure 2E, Figure 3A, 3C). The GUS staining pattern in the secondary vasculature of the pPXC3::GUS plants was similar to that of $p P X Y:: G U S$, except that in PXC3:: GUS, no GUS was not expressed in the interfascicular region (Figure 2E). The GUS staining of the four lines diverged in the roots. GUS was observed in the root tip in the $p P X C 2:: G U S$ line, close to the quiescent center in the pPXC1::GUS and $p P X C 3:: G U S$ lines and in the elongation zone in the $p P X Y:: G U S$ line (Figure 2D) $[13,15,16]$. In contrast to the similarities in the GUS staining patterns in the vascular tissues, there was little similarity between the GUS outside these tissues (i.e. in floral tissues) (Additional file 3).

\section{Expression levels of $P X C 1$ in vascular tissues}

Because the strongest predicted links were between $P X C 1$ and $P X Y / T D R$, we concentrated our efforts on this gene. To further investigate the function of $P X C 1$ in vascular development, transcript levels of $P X C 1$ were determined in parallel with seven known regulators of vascular development in different plant tissues including the leaf lamina (as a control), petiole, young inflorescence stem, old inflorescence stem and hypocotyl. Our data showed that the transcripts for $P X Y$ were most abundant in the xylem fraction of hypocotyls, with levels increasing in older inflorescence stems (Additional file 4C). Expression patterns for HB8, HB15 (HOMEOBOX GENE 15) and NST3 (NAC TRANSCRIPTION FACTOR3) were similar to those observed for $P X Y$ (Additional file $4 \mathrm{~A}, \mathrm{~B}$ and D). $H B 8$ and $H B 15$ are both recognized as molecular markers of procambial cells $[32,33]$ and NST3 encodes a known regulator of secondary cell wall formation in xylem fibers [34,35]. Interestingly, the qRT-PCR data also indicated a similar expression pattern between PXC1 and WOX4, with the highest level of transcript observed in young stem (Additional file 4G and H). CLE41 and CLE44 both encode the B-type CLE peptides that act as the ligands for the TDIF-PXY/TDR signal transduction pathway [31]. As previously reported, the CLE41 transcript was found to be mainly associated with the phloem fraction (Additional file 4E) [13]. This fraction may, however, include some cambium cells as a result of the sample collection (peeling) method used [13]. CLE44 transcript appeared more evenly distributed among the tissue fractions, with a significantly lower level of transcripts in the xylem tissue (Additional file 4F), which is consistent with the recent report of phloem-specific expression of CLE44:: GUS [36]. To summarize the expression patterns described above, a heat map was generated using the qPCR data. Strikingly, the heat map highlighted the similarities between PXC1 and WOX4 in vascular tissues (Figure 4).

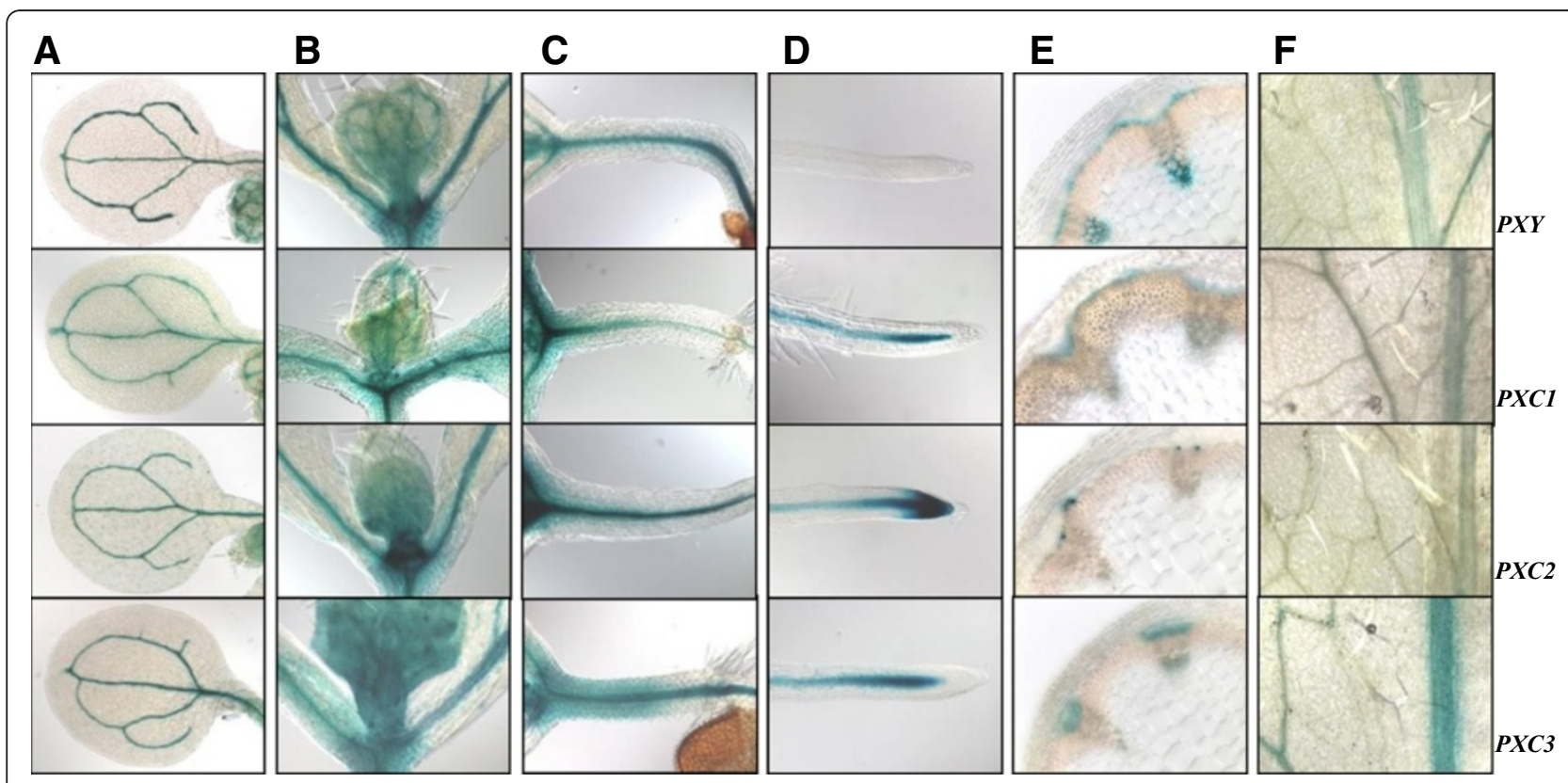

Figure 2 Validation of the microarray data for differential gene expression by GUS staining of transgenic plants harboring $p P X Y:: G U S$, pPXC1::GUS, pPXC2::GUS and pPXC3::GUS. One-week-old plants for a-d, five-week-old plants for e-h and the floral stem was about $15 \mathrm{~cm}$ tall. (A) GUS staining in the cotyledons; (B) GUS staining in the shoot apex; (C) GUS staining in the hypocotyls; (D) GUS staining in the root tip; (E) GUS staining in the stem cross section; (F) GUS staining in the leaves. 


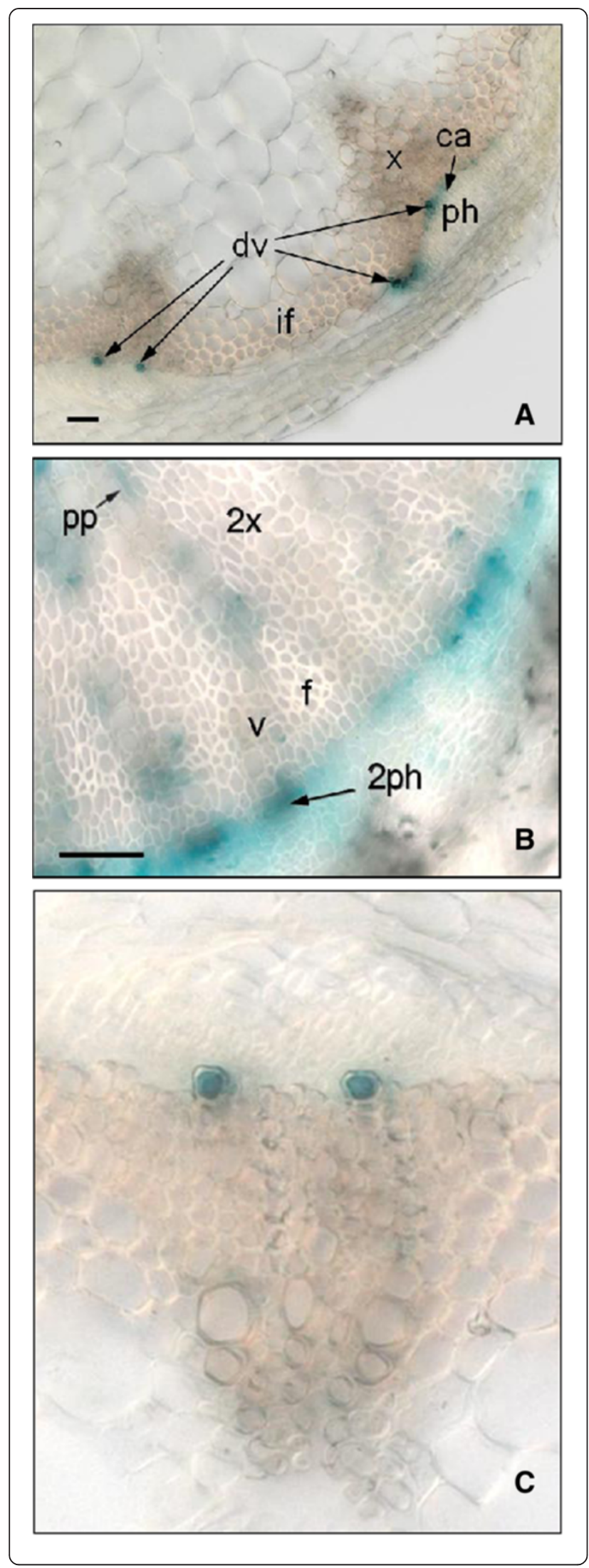

Figure 3 GUS staining in pAtPXC1::GUS and pAtPXC2::GUS transgenic Arabidopsis seedlings. A. Typical GUS staining in basal stem of PAtPXC2::GUS transgenic Arabidopsis seedlings; B. Typical GUS staining in hypocotyl of pAtPXC1::GUS transgenic Arabidopsis seedlings; C. Close-up of A.

\section{Loss of PXC1 function suppresses secondary cell wall formation in xylem fibers}

The $P X C 1$ gene encodes a predicted protein containing a putative kinase domain and an extracellular domain with 21 leucine-rich repeats (eLRR). The predicted PXC1 belongs to the LRR-RLK subfamily III $[2,37]$ along with CLV1 (27\% amino acid identity) and PXY/TDR (28\% amino acid identity). Protein motif search in the Pfam and SMART databases predicted that the kinase domain of PXC1 resembles that of the animal receptor tyrosine kinase domain, which is an unusual characteristic for a plant RLK [26]. Phosphorylation of the soybean homologue of PXC1 (GmLRK1) has been demonstrated to be induced by plant protein extracts, which suggested that some plant proteins may interact with GmLRK1 and phosphorylate it in vivo [26]. Based on the high degree of sequence divergence between PXC1 and other subfamilies of LRR-RLKs, no firm prediction can be made as to its functional role.

Three T-DNA insertion lines were identified for PXC1 in the NASC (the European Arabidopsis Stock Center) mutant collection, including two SALK lines and one WiscDsLox T-DNA line. These three alleles were designated as pxc1-1 (SALK_134974), pxc1-2 (SALK_134975), and $p x c 1-3$ (WiscDsLox470G6), respectively. Both $p x c 1-1$ and $p x c 1-3$ contain insertions in the coding sequence, while $p x c 1-2$ has a T-DNA inserted in the 3'-UTR (Figure 5A). Results of qPCR analyses using a gene specific primer set located in the 3'-UTR indicated that the level of PXC1 mRNA in three mutants was pxc1-1 > pxc1-2 > pxc1-3 (Figure 5B). pxc1-3 displayed only background level of $P X C 1$ expression, suggesting that this allele might represent a null mutant (Figure 5B). Several aspects of plant morphology were affected by the mutation of PXC1. The inflorescence stems of the $p x c 1$ mutants were taller than those of the wild-type, with the phenotype most pronounced in the pxc1-3 line (Additional file 5). By contrast, the inflorescence stem of pxy mutant plants were shorter than the wild-type [16]. When grown under long-day conditions, $p x c 1$ mutants did not show significant difference from the wild-type in terms of cellular morphology as seen from transverse sections of the inflorescence stem (data not shown). Secondary vascular development is enhanced in the inflorescence stems of Arabidopsis when plants are grown under long day conditions (16/8 day/night) and transferred to short-day conditions (8/16 day/night) immediately after bolting. In both wild-type and $p x c 1-2$ 


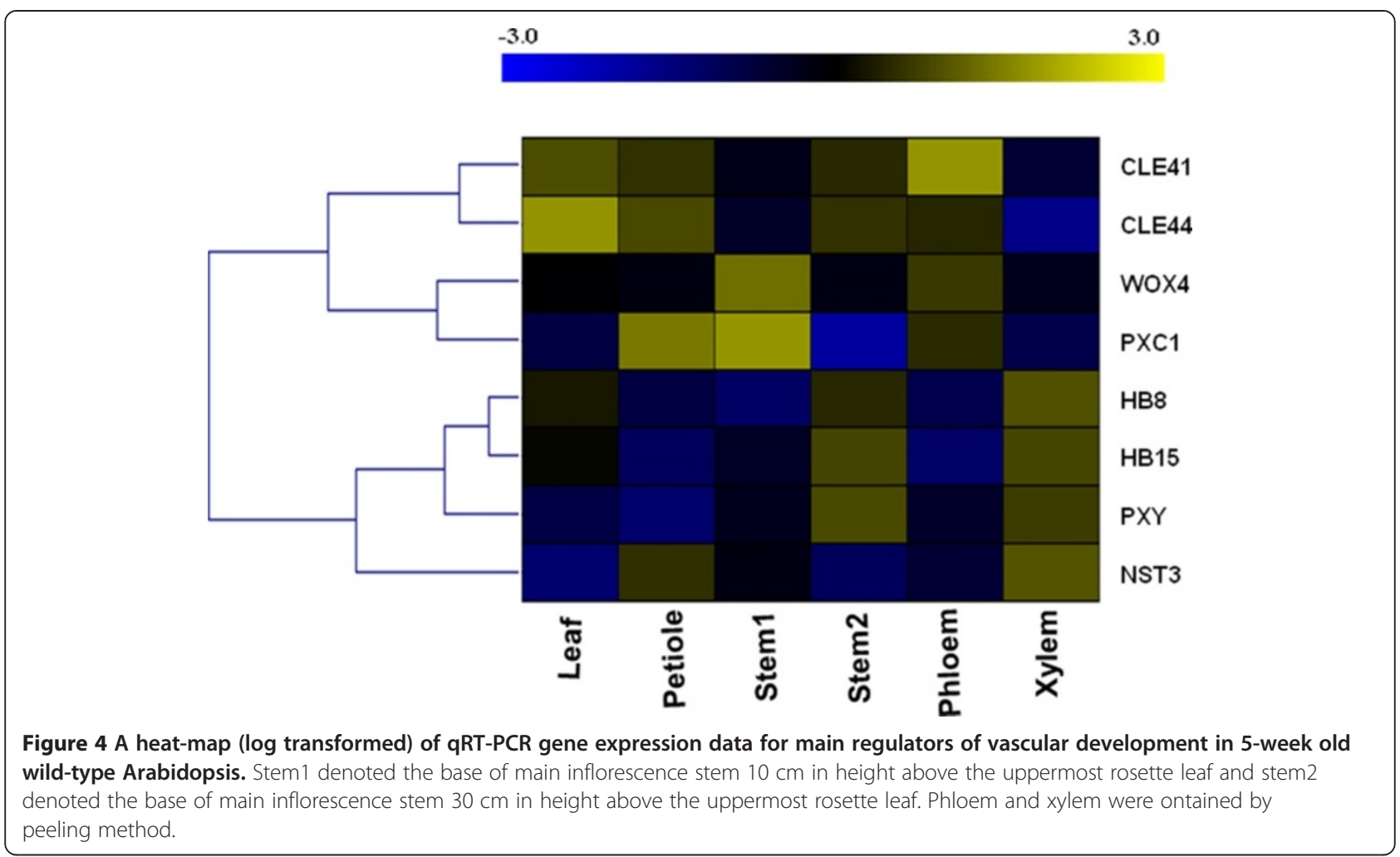

plants, the inflorescence stem grew vertically from this point. In contrast, the stems of $p x c 1-1$ and $p x c 1-3$ plants were unable to support the weight of the continued upright growth of the stem (Figure $5 \mathrm{C}$ ). Ligin staining in cross sections taken from the base of $p x c 1-1, p x c 1-3$ and wild-type inflorescent stems indicated that the two mutant lines were defective in vascular lignification (Figure 5D-L), which then provided an explanation to the inability of the inflorescence stem to support an upright growth. Tissue polarity in the $p x c 1$ mutants appeared to be retained, as opposed to the polarity phenotype in the pxy mutant (Figure 5G-J). Secondary cell wall thicking in fiber cells was also considerably reduced in $p x c 1-1$ plants and absent from $p x c 1-3$ plants (Figure 5I, 5L), indicating a reduced capacity for secondary cell wall synthesis and lignifications in the $p x c 1 \mathrm{mu}-$ tants (Figure 5H, 5K). The vascular bundles of the long day grown plants and those shifted from long to short days wer compared by close-ups in Figure 6 . Interestingly, PXC1 has been identified in the repertoire of genes regulated by $S N D 2$, which is an indirect target of a principal regulator of fiber secondary cell wall formation, SND1 [38,39]. Overexpression of SND2 produced a fiber cell-specific increase in secondary cell wall thickness in Arabidopsis stems and PXC1 was slightly up-regulated in this transgenic line [38]. Thus, the reduced cell wall thickness in the interfascicular fiber cell of $p x c 1-3$ and pxc1-1 mutants indicated that PXC1 is likely playing a role in secondary cell wall formation of fiber cells.

\section{The interactions between PXC1 and TDIF-PXY/TDR} signaling pathway

qPCR analysis of PXY, PXC1, CLE41, CLE44 and WOX4 expression was analyzed in the $p x y / t d r, p x c 1, c l e 41$, cle 44 and wox4 mutants. Transcript abundances of $P X Y$ and CLE41 were not dramatically affected in the $p x y / t d r, p x c 1$ and wox4 knockout lines (Figure 7A). The transcript level of PXC1, WOX4 and CLE44 were significantly increased in $p x y, p x c 1$ and wox4 mutants compared to the wild-type (Figure 7A). The elevated expression level of WOX4 in the pxy background was unexpected since that WOX4 is a key downstream target of the TDIF-PXY/TDR signaling pathway [13]. This result indicated a possibility that PXY might not be the only receptor acting upstream of WOX4. Meanwhile, instead of young seedlings [13], 5-week-old hypocotyls, which contain more secondary growth, were used in this study and the implication of PXY/TDR in xylem development has not been investigated in details. The dramatic increase in PXC1 transcripts occurred in the $p x y$ and wox4 knockout mutants indicated that either the PXY-WOX4 pathway negatively regulate the expression of $P X C 1$, or that $P X C 1$ is upregulated in these mutants as a compensatory measure (Figure 7A). These data also suggested that the signal transduction pathways mediated by 


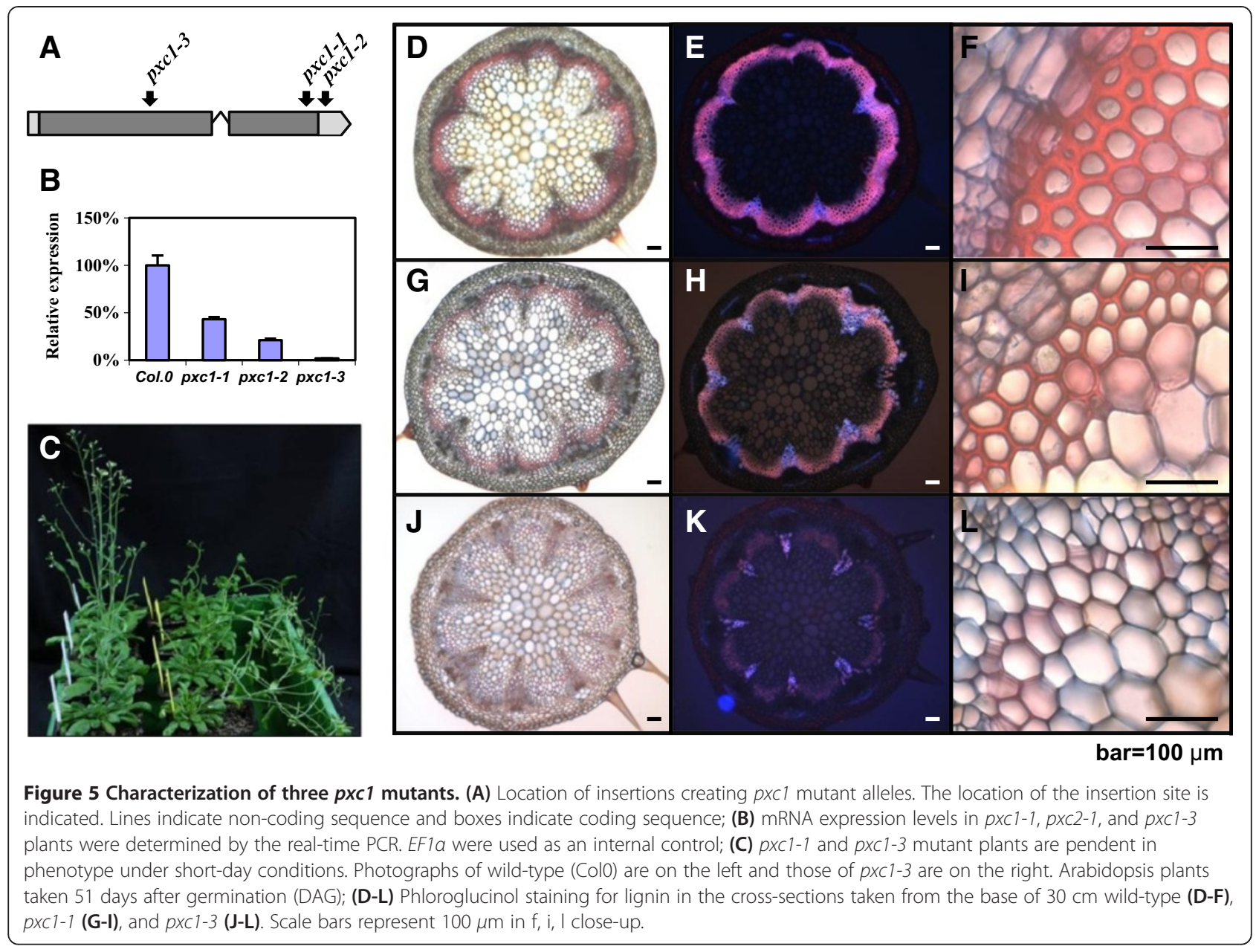

PXY and PXC1 are at least partly overlapping. However, it is hard to specify the position of PXC1 relative to the TDIF/PXY/WOX4 pathway because we could not judge which alterations in gene expression resulting from forward responses or feedback responses.

As opposed to the expression profiles of plants grown under long-day conditions, the short-day shift experiment induced a strong decrease in PXY/TDR and WOX4 transcript levels in the inflorescence stems of the $p x c 1$ mutants, particularly in the pxc1-3 line (Figure 7B). The reduced capacity to produce an interfascicular cambium in the pxc1 mutants may explain the reduction in $P X Y /$ $T D R$ and WOX4 transcript levels, or vice versa, the reduced expression of PXY/TDR and WOX4 may be the underlying cause of the lack of interfascicular cambium. Future research will attempt to answer this question. It has been reported that the exogenous application of the TDIF/CLE41/44 peptide ligand resulted in an increase in the transcript levels of $H B 8, H B 15, W O X 4$ and $P X Y$ genes [40]. We similarly over-expressed CLE44 in Arabidopsis and analyzed its effects on PXC1 transcript levels. Three CLE44ox lines exhibiting from 65 to 85fold increases in CLE44 transcript levels compared to wild-type level were identified and analyzed (Figure 7C). These lines exhibited similar phenotypes to the CLE41ox and CLE42ox lines reported previously [13,31]. Transcripts for PXC1, PXY/TDR, WOX4, HB-8 and REV were elevated compared to wild-type plants in all three of our CLE44ox lines (Figure 7D). Once again, the explanation may be that the changes in gene expression are the result of developmental abnormalities. That is to say that the affected genes might not be directly regulated by CLE44 over-expression, but the result of changes in cellular composition, such as an increase in the abundance of dividing/undifferentiated cells in the transgenic line (Figure 7D). However, the similarities between PXC1 and elements in the TDIF-PXY/ $T D R$ pathway indicate that $\mathrm{PXC1}$ functions synergistically with the TDIF-PXY/TDR signaling pathway.

\section{Conclusions}

LRR-RLK receptors have been shown to mediate multiple signal transduction pathways. It is clear from work in the 

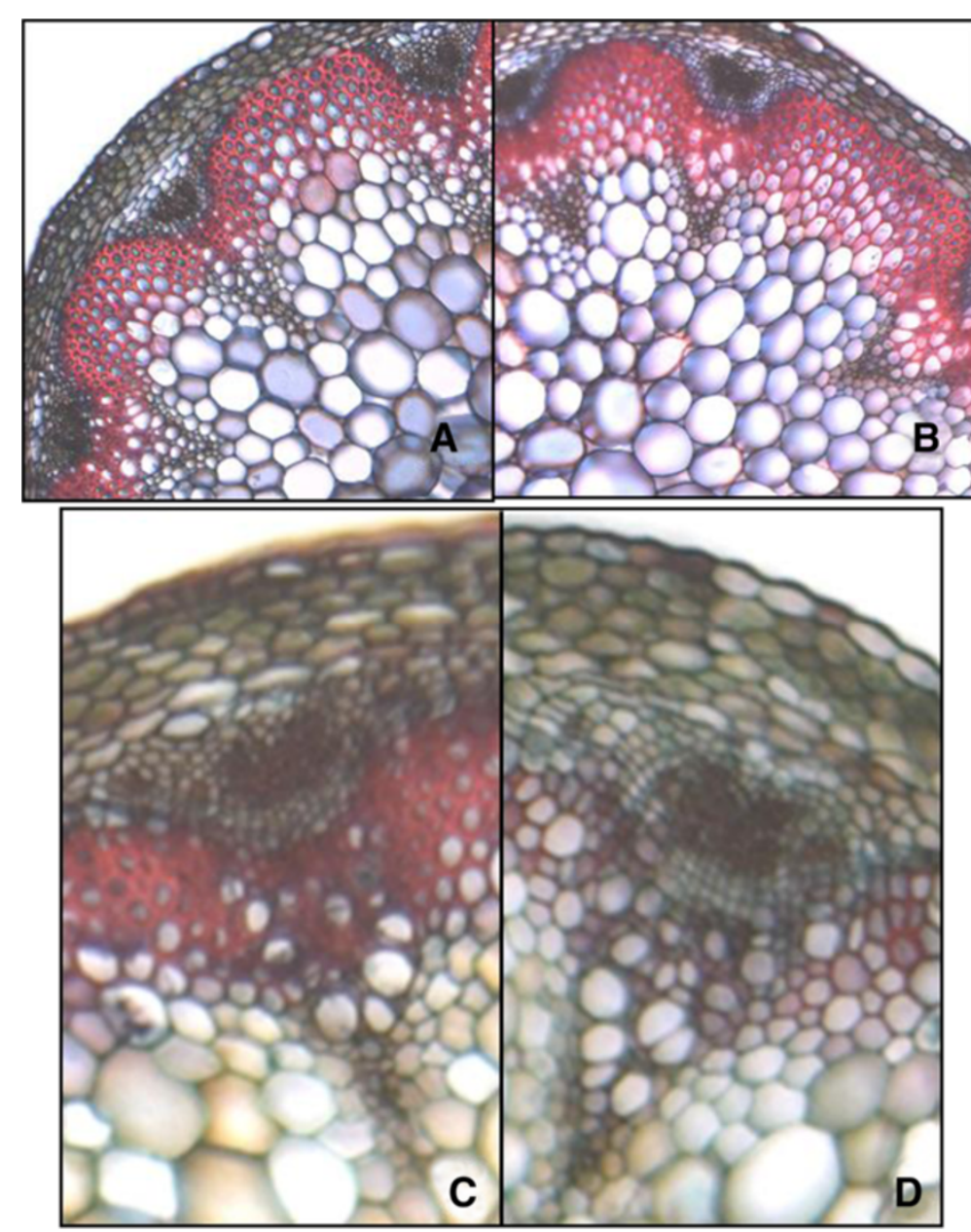

Figure 6 Phloroglucinol staining for lignin in the cross-sections taken from $30 \mathrm{~cm}$ above the base of wild-typeand pxc1-3 before and after long-day to short-day transition. (A) Wild-type Arabidopsis plant grown in long-day conditions; (B) Arabidopsis pxc1-3 plant grown in long-day conditions; (C) Wild-type Arabidopsis plant grown in short-day conditions after bolting; (D) Arabidopsis pxc1-3 plant grown in short-day conditions after bolting.

SAM that the combined actions of multiple LRR-RLKs, each with defined functions, are required to maintain the balance between stem cell division and differentiation in meristems. In vascular tissue, TDIF-PXY/TDR signal transduction pathway plays multiple roles in xylem development including the promotion of cambial cell division and repression of xylogenesis [31]. In this work, from in silico analyses, a new LRR-RLK component involved in the regulation of plant vasculature development, PXC1, was introduced with its expression patterns correlated to that of $P X Y$ gene. PXC1 probably plays its roles in a regulatory network which also incorporates the PXY/TDRWOX4 signaling pathway and regulates the maturation of interfascicular fiber cells. The co-regulation network suggested that the loss of $P X C 1$ function might retard the initiation of secondary cell wall deposition by prolonging the course of cell wall remodeling and reorganization during the procedure of cell expansion.

\section{Materials and methods}

\section{Expression profiling, co-expression analyses and gene} functional clustering

Microarray expression data sets were explored for the predicted AtLRR-RLK genes using the Arabidopsis Affymetrix GeneChip average data available on the GENEVESTIGATOR analysis tool site (http://www.genevestigator.ethz.ch) [20]. The Gene Co-expression Analysis (GeneCAT) Toolbox at http://genecat.mpg.de/cgi-bin/Ainitiator.py [41] was used to generate the Expression Tree which clustered genes by the similarity of their expression profiles and visualized those similarities using a dendogram. To minimize the effects of experimental artifacts, data were renormalized, 

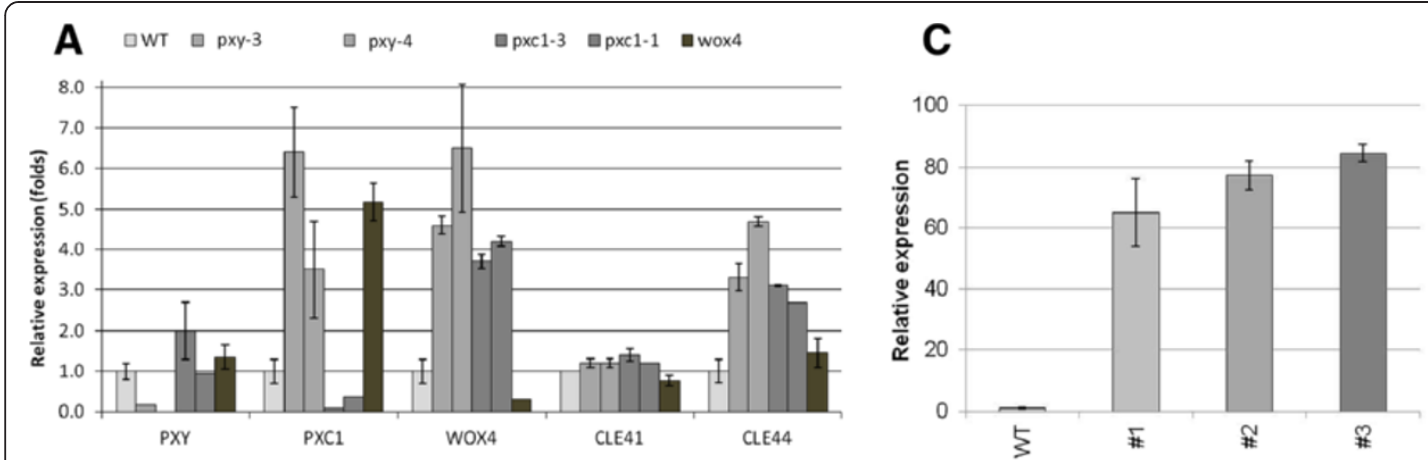

B
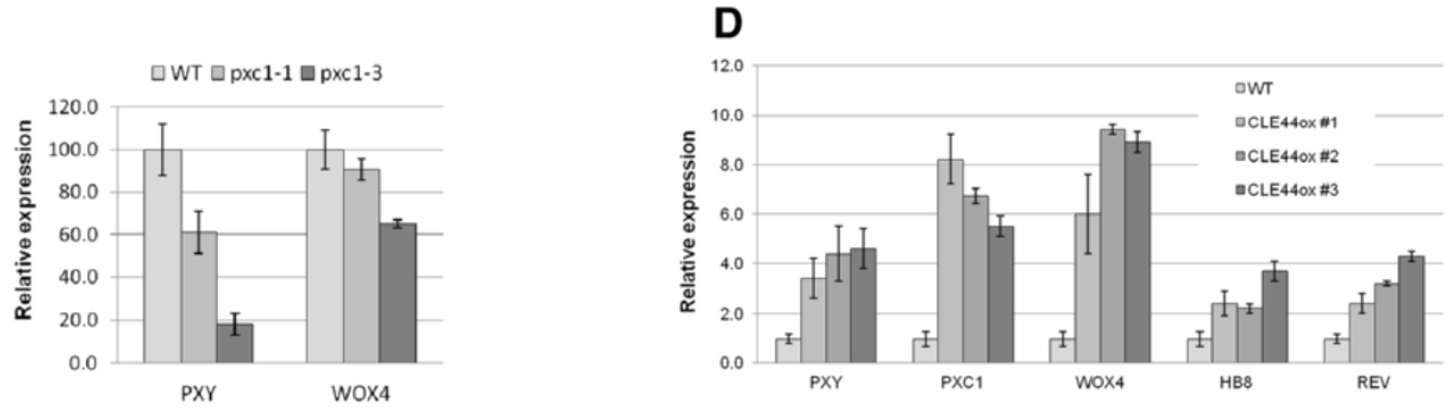

Figure 7 Transcript level changes of genes involved in TDIF/PXY/WOX4 pathway and vascular development markers in wild-type Arabidopsis and various mutants and overexpressors. (A) Gene expression levels analyzed by qPCR in the hypocotyls of 5-week-old wild-type Arabidopsis and mutants under long-day conditions; (B) Change in gene expression level in the basal stem part of two 51-day-old PXC1 mutants after the long-day to short-day shifting experiment; (C) Relative CLE44 expression level measured by qRT-PCR in Col-0 and three CLE44 ox plants with inflorescence stems as tall as $15 \mathrm{~cm}$; (D) Relative expression level of marker genes in wild-type and three CLE44 ox lines with inflorescence stems as tall as $15 \mathrm{~cm}$.

and Pearson's correlation coefficient between genes was weighted in ATTED-II [28].

\section{Identification of loss-of-function mutants and construction of transgenic Arabidopsis}

Seeds for segregating T3 plants harboring the $p x c 1$ alleles in the Col-0 ecotype background were obtained from the NASC (Nottingham Arabidopsis Stock Centre. The location of the T-DNA insertion was determined by sequencing and homozygous lines were identified by PCR. For the production of the 35Spro::CLE44 construct, the coding sequence of Arabidopsis CLE44 was amplified from genomic DNA, cloned into pDONR201 vector (Invitrogen), and subcloned into the $\mathrm{pK} 2 \mathrm{GW} 7$ vector using the Gateway cloning system (Invitrogen). Native promoter::GUS-GFP fusion constructs were made for $P X Y$ and $P X C 1 / 2 / 3$ by cloning the amplified promoter regions into the binary vector pKGWFS7 $^{\mathrm{m}}$ [42] via pDONR201 (Invitrogen). Vectors were then transformed into Agrobacterium tumefaciens strain GV3101 (pMP90). Arabidopsis plants were transformed using the floral dip method [44]. Positive transgenic Arabidopsis plants were selected based on kanamycin resistance conferred by the T-DNA. T2 seeds from at least 24 independent positive lines for each construct were harvested for expression analyses.

\section{Histochemistry}

Plant tissues at various developmental stages were vacuuminfiltrated for $2 \mathrm{~min}$ in GUS solution including $1 \mathrm{mM}$ $\mathrm{X}$-gluc, $50 \mathrm{mM}$ sodium phosphate ( $\mathrm{pH}$ 7.0), 0.1\% Triton $\mathrm{X}-100,1 \mathrm{mM}$ potassium ferricyanide and $1 \mathrm{mM}$ potassium ferrocyanide, and incubated at $37^{\circ} \mathrm{C}$ overnight. Destaining of the samples were performed by incubation in $0.24 \mathrm{M}$ $\mathrm{HCl}$ and $20 \% \mathrm{MeOH}$ solution at $55^{\circ} \mathrm{C}$ for $15 \mathrm{~min}$, then in $7 \% \mathrm{NaOH}$ and $60 \% \mathrm{EtOH}$ solution at room temperature for $15 \mathrm{~min}$. The samples were dehydrated through ethanol series (40\%, 20\%, and $10 \%$ in water). For sectioning, samples were embedded in $4 \%(\mathrm{w} / \mathrm{v})$ agar and sectioned at $50 \mu \mathrm{m}$ with a vibratome (Leica vt1000s). Samples were then mounted in glycerol and analyzed by bright field transmitted microscopy using an Axioplan 2 microscope (Carl Zeiss Inc. Thornwood, NY, USA). Images were captured by AxioCam HRc and Axiovision software (AxionVs40 V4.5.0.0).

\section{Safranin/alcian-blue staining}

Plants were grown under long day conditions and then were moved into short day conditions right after bolting. Long day conditions were $16 \mathrm{~h}$ light $/ 8 \mathrm{~h}$ dark, $75 \%$ humidity, $150 \mu \mathrm{E}$ of irradiance and short day conditions were $8 \mathrm{~h}$ light $/ 16 \mathrm{~h}$ dark, $75 \%$ humidity, $150 \mu \mathrm{E}$ of irradiance. For 
sectioning, samples were embedded in $4 \%(\mathrm{w} / \mathrm{v})$ agar, and sectioned at $50 \mu \mathrm{m}$ with a vibratome (Leica vt1000s). Sections were then stained in one part safranin $(1 \% \mathrm{w} / \mathrm{v}$ safranin in $50 \%$ ethanol) and two parts alcian-blue $(1 \% \mathrm{v} / \mathrm{w}$ alcian-blue, $1 \% \mathrm{v} / \mathrm{v}$ formalin, $36 \%$ formaldehyde) in $0.05 \%$ glacial acetic acid. The sections were subsequently rinsed in $\mathrm{ddH}_{2} \mathrm{O}$ and mounted in $50 \%$ glycerol. Slide-mounted sections were viewed using a Zeiss Axioplan 2 compound microscope with a Zeiss AxioCamHRc digital camera (Carl Zeiss, Inc., Thornwood, NY, USA).

\section{Gene expression analyses by quantitative PCR}

All samples came from five-week-old wild type plants under long-day conditions when the inflorescence stems reached a height of $25 \mathrm{~cm}$, except that stem 1 was from younger plants whose inflorescence was $10 \mathrm{~cm}$ high. Fully expanded leaves were harvested without the midrib, and petioles were harvested from the fully expanded leaves. Stem 1 (younger stem) and stem2 (older stem) were both from the basal part of the inflorescence stems at $1-5 \mathrm{~cm}$ above the rosette. All nodes were removed from the stem samples. The phloem and xylem samples were obtained simply by separating the bark/phloem from the xylem core. Total RNA $(0.2 \mu \mathrm{g})$ was used for cDNA synthesis using the Thermoscript RT-PCR kit (Invitrogen Life Technologies, USA). Real-time qPCR was performed with the LightCycler instrument (Roche Diagnostics, Germany). Each mRNA value was corrected by the measurements obtained in the same sample for $18 \mathrm{~S}$ mRNA and elongation factor 1 (EF1 $\alpha)$ using Delta Delta method [44]. The primer sequences utilized in this study were listed in Additional file 6. Each amplification included three technical replicates and their results were averaged to give the value for a single biological replicate. Three biological replicates were prepared for each treatment using material harvested from 10-15 plants in each case. The means of relative expression for each sample were examined using one-way analysis of variance (ANOVA) method (significance at $P<0.05$ ).

\section{Additional files}

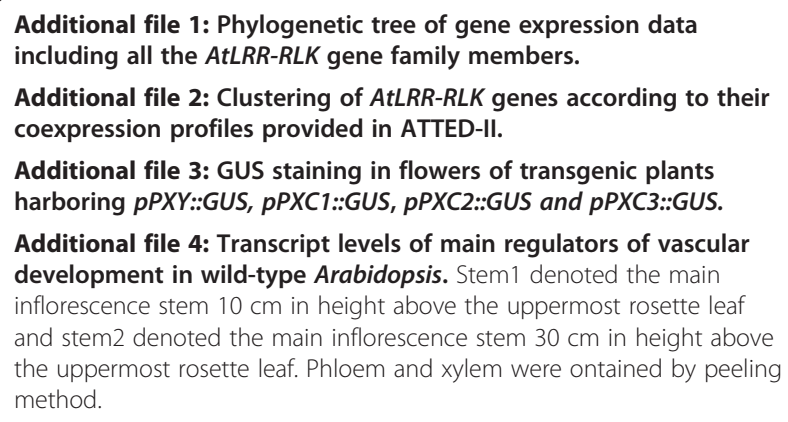

Additional file 5: Inflorescence height ( $\mathrm{mm})$ in WT, pxc1-1 and pxc1-3 mutants after bolting.

Additional file 6: Primers for qRT-PCR in the current study.

\section{Competing interests}

The authors declare that they have no competing interests.

\section{Author's contributions}

JW, MK, LZ, PC and DD performed the experimental work. BZ, ON and GS designed and coordinated the project. JW, BZ and BJ wrote the paper. All authors read and approved the final manuscript.

\section{Acknowledgements}

This study was supported by National Program on Key Basic Research Project (2012CB1 14500), National High Technology Research and Development Program (2011AA100200) and National Natural Science Foundation (31270644, 31170564 and 31070600) of China. It was also supported by Swedish Foundation for Strategic Research.

\section{Author details}

'School of Environmental Science and Engineering, Tianjin University, 300072 Tianjin, China. ${ }^{2}$ Umeå Plant Science Centre, Department of Forest Genetics and Plant Physiology, Swedish University of Agricultural Sciences, SE-901 83 Umeå, Sweden. ${ }^{3}$ College of Plant Science and Technology, Huazhong Agricultural University, 430070 Wuhan, China. ${ }^{4}$ Umeå Plant Science Centre, Department of Plant Physiology, Umeå University, SE-901 87 Umeå, Sweden. ${ }^{5}$ Faculty of Agriculture and Environment, Department of Plant and Food Sciences, University of Sydney, Sydney, Australia. ${ }^{6}$ College of Horticulture and Forestry Sciences, Huazhong Agricultural University, 430070 Wuhan, China.

Received: 6 February 2013 Accepted: 25 June 2013

Published: 1 July 2013

\section{References}

1. Ten Hove CA, Bochdanovits Z, Jansweijer VM, Koning FG, Berke L, SanchezPerez GF, Scheres B, Heidstra R: Probing the roles of LRR RLK genes in Arabidopsis thaliana roots using a custom T-DNA insertion set. Plant Mol Biol 2011, 76(1-2):69-83.

2. Shiu SH, Bleecker AB: Receptor-like kinases from Arabidopsis form a monophyletic gene family related to animal receptor kinases. Proc Natl Acad Sci USA 2001, 98(19):10763-10768.

3. Afzal AJ, Wood AJ, Lightfoot DA: Plant receptor-like serine threonine kinases: roles in signaling and plant defense. Mol Plant Microbe Interact 2008, 21(5):507-517.

4. Clark SE, Running MP, Meyerowitz EM: CLAVATA1, a regulator of meristem and flower development in Arabidopsis. Development 1993, 119(2):397-418.

5. Clark SE, Williams RW, Meyerowitz EM: The CLAVATA1 gene encodes a putative receptor kinase that controls shoot and floral meristem size in Arabidopsis. Cell 1997, 89:575-585.

6. Schoof H, Lenhard M, Haecker A, Mayer KFX, Jurgens G, Laux T: The stem cell population of Arabidopsis shoot meristems is maintained by a regulatory loop between the CLAVATA and WUSCHEL genes. Cell 2000, 100(6):635-644.

7. Brand $U$, Grunewald M, Hobe M, Simon R: Regulation of CLV3 expression by two homeobox genes in Arabidopsis. Plant Physiol 2002, 129(2):565-575.

8. Sarkar AK, Luijten M, Miyashima S, Lenhard M, Hashimoto T, Nakajima K, Scheres B, Heidstra R, Laux T: Conserved factors regulate signalling in Arabidopsis thaliana shoot and root stem cell organizers. Nature 2007, 446(7137):811-814.

9. Stahl $Y$, Wink RH, Ingram GC, Simon R: A signaling module controlling the stem cell niche in Arabidopsis root meristems. Curr Biol 2009, 19(11):909-914.

10. Brand U, Fletcher JC, Hobe M, Meyerowitz EM, Simon R: Dependence of stem cell fate in Arabidopsis on a feedback loop regulated by CLV3 activity. Science 2000, 289(5479):617-619.

11. Mayer KFX, Schoof H, Haecker A, Lenhard M, Jurgens G, Laux T: Role of WUSCHEL in regulating stem cell fate in the Arabidopsis shoot meristem. Cell 1998, 95(6):805-815. 
12. DeYoung BJ, Bickle KL, Schrage KJ, Muskett P, Patel K, Clark SE: The CLAVATA1-related BAM1, BAM2 and BAM3 receptor kinase-like proteins are required for meristem function in Arabidopsis. Plant J 2006, 45(1):1-16.

13. Hirakawa $Y$, Shinohara $H$, Kondo $Y$, Inoue A, Nakanomyo I, Ogawa M, Sawa S, Ohashi-Ito K, Matsubayashi Y, Fukuda H: Non-cell-autonomous control of vascular stem cell fate by a CLE peptide/receptor system. Proc Natl Acad Sci USA 2008, 105(39):15208-15213.

14. Breuninger $H$, Rikirsch $E$, Hermann M, Ueda M, Laux T: Differential expression of WOX genes mediates apical-basal axis formation in the Arabidopsis embryo. Dev Cell 2008, 14(6):867-876.

15. Hirakawa $Y$, Kondo $Y$, Fukuda H: TDIF peptide signaling regulates vascular stem cell proliferation via the WOX4 homeobox gene in Arabidopsis. Plant Cell 2010, 22(8):2618-2629.

16. Fisher $\mathrm{K}$, Turner S: PXY, a receptor-like kinase essential for maintaining polarity during plant vascular-tissue development. Curr Biol 2007, 17(12):1061-1066.

17. Strabala TJ, O'Donnell PJ, Smit AM, Ampomah-Dwamena C, Martin EJ, Netzler N, Nieuwenhuizen NJ, Quinn BD, Foote HC, Hudson KR: Gain-of-function phenotypes of many CLAVATA3/ESR genes, including four new family members, correlate with tandem variations in the conserved CLAVATA3/ESR domain. Plant Physiol 2006, 140(4):1331-1344.

18. Whitford R, Fernandez A, De Groodt R, Ortega E, Hilson P: Plant CLE peptides from two distinct functional classes synergistically induce division of vascular cells. Proc Natl Acad Sci USA 2008, 105(47):18625-18630.

19. Yaginuma $H$, Hirakawa $Y$, Kondo $Y$, Ohashi-lto $K$, Fukuda H: A novel function of TDIF-related peptides: promotion of axillary bud formation. Plant Cell Physiol 2011, 52(8):1354-1364.

20. Zimmermann P, Hirsch-Hoffmann M, Hennig L, Gruissem W: GENEVESTIGATOR. Arabidopsis microarray database and analysis toolbox. Plant Physiol 2004, 136(1):2621-2632.

21. Agusti J, Lichtenberger R, Schwarz M, Nehlin L, Greb T: Characterization of transcriptome remodeling during cambium formation identifies MOL1 and RUL1 as opposing regulators of secondary growth. PLOS Genet 2011 7(2):e1001312

22. Clay NK, Nelson T: VH1, a provascular cell-specific receptor kinase that influences leaf cell patterns in Arabidopsis. Plant Cell 2002, 14(11):2707-2722

23. Sottosanto JB, Gelli A, Blumwald E: DNA array analyses of Arabidopsis thaliana lacking a vacuolar $\mathrm{Na}+\mathrm{H}+$ antiporter: impact of AtNHX1 on gene expression. Plant J 2004, 40(5):752-771.

24. Loreti E, Poggi A, Novi G, Alpi A, Perata P: A genome-wide analysis of the effects of sucrose on gene expression in Arabidopsis seedlings under anoxia. Plant Physiol 2005, 137(3):1130-1138.

25. Krinke O, Ruelland E, Valentova O, Vergnolle C, Renou JP, Taconnat L, Flem M, Burketova L, Zachowski A: Phosphatidylinositol 4-kinase activation is an early response to salicylic acid in Arabidopsis suspension cells Plant Physiol 2007, 144(3):1347-1359.

26. Kim S, Kim SJ, Shin YJ, Kang JH, Kim MR, Nam KH, Lee MS, Lee SH, Kim YH, Hong SK, et al: An atypical soybean leucine-rich repeat receptor-like kinase, GmLRK1, may be involved in the regulation of cell elongation. Planta 2009, 229(4):811-821.

27. Obayashi T, Kinoshita K, Nakai K, Shibaoka M, Hayashi S, Saeki M, Shibata D, Saito K, Ohta H: ATTED-II: a database of co-expressed genes and cis elements for identifying co-regulated gene groups in Arabidopsis. Nucleic Acids Res 2007, 35(Database issue):D863-D869.

28. Obayashi T, Hayashi S, Saeki M, Ohta H, Kinoshita K: ATTED-II provides coexpressed gene networks for Arabidopsis. Nucleic Acids Res 2009, 37(Database issue):D987-D991.

29. Baima S, Nobili F, Sessa G, Lucchetti S, Ruberti I, Morelli G: The expression of the Athb-8 homeobox gene is restricted to provascular cells in Arabidopsis thaliana. Development 1995, 121(12):4171-4182.

30. Otsuga D, DeGuzman B, Prigge MJ, Drews GN, Clark SE: REVOLUTA regulates meristem initiation at lateral positions. Plant J 2001, 25(2):223-236

31. Etchells JP, Turner SR: The PXY-CLE41 receptor ligand pair defines a multifunctional pathway that controls the rate and orientation of vascular cell division. Development 2010, 137(5):767-774.
32. Baima S, Nobili F, Sessa G, Lucchetti S, Ruberti I, Morelli G: The expression of the ATHB-8 homeobox gene is restricted to provascular cells in Arabidopsis thaliana. Development 1995, 121:4171-4182.

33. Ohashi-Ito K, Fukuda H: HD-zip III homeobox genes that include a novel member, ZeHB-13 (Zinnia)/ATHB-15 (Arabidopsis), are involved in procambium and xylem cell differentiation. Plant Cell Physiol 2003, 44(12):1350-1358.

34. Mitsuda N, Iwase A, Yamamoto H, Yoshida M, Seki M, Shinozaki K, OhmeTakagi M: NAC transcription factors, NST1 and NST3, are key regulators of the formation of secondary walls in woody tissues of Arabidopsis. Plant Cell 2007, 19(1):270-280.

35. Mitsuda N, Ohme-Takagi M: NAC transcription factors NST1 and NST3 regulate pod shattering in a partially redundant manner by promoting secondary wall formation after the establishment of tissue identity. Plant J 2008, 56(5):768-778.

36. Malinowski R, Smith JA, Fleming AJ, Scholes JD, Rolfe SA: Gall formation in clubroot-infected Arabidopsis results from an increase in existing meristematic activities of the host but is not essential for the completion of the pathogen life cycle. Plant J 2012, 71(2):226-238.

37. Shiu SH, Karlowski WM, Pan R, Tzeng YH, Mayer KF, Li WH: Comparative analysis of the receptor-like kinase family in Arabidopsis and rice. Plant Cell 2004, 16(5):1220-1234.

38. Hussey SG, Mizrachi E, Spokevicius AV, Bossinger G, Berger DK, Myburg AA: SND2, a NAC transcription factor gene, regulates genes involved in secondary cell wall development in Arabidopsis fibres and increases fibre cell area in Eucalyptus. BMC Plant Biol 2011, 11:173.

39. Zhong R, Demura T, Ye ZH: SND1, a NAC domain transcription factor, is a key regulator of secondary wall synthesis in fibers of Arabidopsis. Plant Cell 2006, 18(11):3158-3170.

40. Hirakawa $Y$, Kondo $Y$, Fukuda $\mathrm{H}$ : Regulation of vascular development by CLE peptide-receptor systems. J Integr Plant Biol 2010, 52(1):8-16.

41. Mutwil M, Obro J, Willats WGT, Persson S: GeneCAT - novel webtools that combine BLAST and co-expression analyses. Nucleic Acids Res 2008, 36:W320-W326.

42. Karimi M, Inze D, Depicker A: GATEWAY vectors for Agrobacteriummediated plant transformation. Trends Plant Sci 2002, 7(5):193-195.

43. Clough SJ, Bent AF: Floral dip: a simplified method for Agrobacteriummediated transformation of Arabidopsis thaliana. Plant $J 1998$ 16(6):735-743.

44. Pfaffl MW: A new mathematical model for relative quantification in real-time RT-PCR. Nucleic Acids Res 2001, 29(9):e45

doi:10.1186/1471-2229-13-94

Cite this article as: Wang et al:: The Arabidopsis LRR-RLK, PXC1, is a regulator of secondary wall formation correlated with the TDIF-PXY /TDR-WOX4 signaling pathway. BMC Plant Biology 2013 13:94.

\section{Submit your next manuscript to BioMed Central and take full advantage of:}

- Convenient online submission

- Thorough peer review

- No space constraints or color figure charges

- Immediate publication on acceptance

- Inclusion in PubMed, CAS, Scopus and Google Scholar

- Research which is freely available for redistribution 